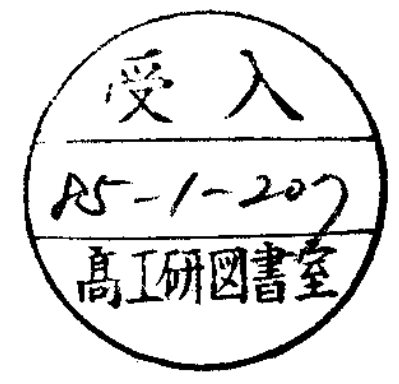

\title{
JET STRUCTURE IN HIGH MASS DIFFRACTIVE SCATTERING
}

\author{
G. Ingelman \\ CERN, CH-1211 Geneva 23, Switzerland \\ P.E. Schlein \\ University of California ${ }^{*}$ ), Los Angeles, CA 90024, USA
}

\section{A B S T R A C T}

We suggest that high- $p_{t}$ jets may emerge from diffractively produced high mass states. Experimental measurements of such high-p structure would give new and valuable insight about the nature of the exchanged pomeron, or pomeron-like object. With the assumption of an effective gluon distribution for the pomeron structure, we estimate the cross-section for the process $\bar{p}+p \rightarrow \bar{p}+X$, where $X$ contains two high- $p_{t}$ jets. Observable rates are found at SPS and Fermilab collider energies.

*) Supported by U.S. National Science Foundation Grant PHY79/24766. 
In hadronic interactions it is possible to produce quite massive systems via diffractive excitation. In the diffractive process

$$
\bar{p}+p \rightarrow \bar{p}+X
$$

the mass, $M_{X}$, of the system $X$ is related to Feynman- $x_{F}$ of the recoil antiproton by $M_{X}^{2}=\left(1-x_{F}\right) \cdot s$. Bozzo et al. [1] have recently shown that the gross features of this process, namely scaling of the invariant cross-section and $d \sigma / d M_{X}^{2} \sim 1 / M_{X}^{2}$ at fixed $t$, are valid up $v_{s}=540$ $\mathrm{GeV}$ at the CERN SPS collider. However, very little is known about the stucture of the system $X$.

For large $x_{F}$ the reaction (1) is believed to proceed via the exchange of a pomeron, as depicted in Fig. 1a.'Although this phenomenological description agrees rather well with existing data $[2,3]$, the true nature of the pomeron is still mysterious. It has been suggested [4] that the pomeron may be a system of two or more gluons, but there is no direct experimental evidence for a partonic structure. One might expect the pomeron to have typical hadronic properties so that the system $X$, produced in a pomeron-proton collision, would resemble normal minimum bias events in hadron-hadron scattering at the corresponding invariant mass, $M_{X}$.

It may be possible to probe the exchanged pomeron (or pomeronlike object) in a hard scattering process as, for example, between a gluon in the pomeron and a parton in the proton (Fig. 1b). This would give rise to a very characteristic event topology, as illustrated in Fig. 1c, with a quasi-elastically scattered antiproton and an opposite (in rapidity) system having two high- $p_{t}$ jets ( $p_{t}$ with respect to the beam axis) and two low- $p_{t}$ spectator jets from the noninteracting partons in the pomeron and proton respectively.

The process we have in mind thus proceeds in two steps as shown in Fig. 1a. First, a pomeron is emitted from the antiproton at the upper vertex with a momentum transfer, $t$. In the second step this pomeron interacts with the proton in a large momentum transfer process between constituent partons. Based on the success of pomeron phenomenology for diffractive dissociation and elastic scattering, we assume this type of factorisation to be a good approximation. Although the pomeron is thought to interact coherently in the dominant low momentum transfer processes, in the present calculation we assume an individual coupling of its gluon constituent to 
a quark or gluon in the proton at the lower vertex. This should be justified because of the large momentum transfer at that vertex.

In order to make cross-section estimates for this process, we assume in this Letter that such high-p $p_{t}$ events are mediated by the same exchange as in ordinary diffractive events. Factorisation allows us to express the cross-section for the diffractive two-jet events in terms of the observed single diffractive cross-section, $\sigma_{s d}$;

$$
\frac{d^{2} \sigma_{j j}}{d t d M_{x}^{2}}=\frac{d^{2} \sigma_{s d}}{d t d M_{x}^{2}} \cdot \frac{\sigma_{p P \rightarrow j j}}{\sigma_{p P \rightarrow X}}
$$

We first discuss the proton-pomeron hard scattering cross-section, $\sigma_{\mathrm{p} \mathbf{P} \rightarrow \mathrm{jj}}$, and then the proton-pomeron total cross-section, $\sigma_{\mathrm{P} \mathbf{P} \rightarrow \mathrm{X}}$.

With the assumption that the pomeron has a gluonic substructure, the cross-section $\sigma_{p \mathbb{P} \rightarrow j j}($ Fig. $1 b$ ) can be calculated as

$$
\sigma_{p \mathbb{P} \rightarrow j j}\left(M_{x}\right)=\int d x_{1} d x_{2} d \hat{t} \sum_{i, k} f_{i}\left(x_{1}, Q^{2}\right) G\left(x_{2}\right) \frac{d \hat{\sigma}_{i}^{k}}{d \hat{t}}
$$

where $G(x)$ is the effective pomeron structure function, $d \hat{\sigma}_{i}^{k} / d \hat{t}$ are standard QCD matrix elements [5] and the summations go over all possible parton-parton scattering subprocesses. We use a standard $\mathrm{Q}^{2}$-dependent parametrisation [6] for the proton structure functions, $f_{i}$.

Eq. (3) is evaluated for the following two structure functions

$$
\begin{aligned}
& x G(x)=6 \cdot x \cdot(1-x)^{1} \\
& x G(x)=6 \cdot(1-x)^{5}
\end{aligned}
$$

which represent two extreme possibilities. The first distribution, which is based on counting-rule ideas assuming that two gluons share the pomeron momentum (hence the symmetry under $x \rightarrow 1-x$, and $\langle x\rangle=1 / 2)$, may be unrealistically hard if $Q C D Q^{2}$-evolution occurs or if the pomeron were a many-gluon system. This latter case is better represented by Eq. (5), which has $\langle x\rangle=1 / 7$ and resembles the gluon structure of the proton. In both cases the distributions are normalised such that the whole pomeron momentum is carried by gluons, i.e. $\int_{0}^{1} x G(x) d x=1$. One could argue that a quark- 
antiquark sea should be present also but this would only make small quantitative changes in the results below and we therefore choose to neglect this possibility in order not introduce further complications at this stage. We also neglect for the time being any $\mathrm{Q}^{2}$ dependence of the pomeron structure function. Such a logarithmic dependence should in any case be much smaller than the difference between the two assumed structure functions.

We further assume that the gluon distribution does not depend on $t$, the pomeron invariant mass. There is no a priori knowledge on this point, but in analogy with the structure function of the photon [7] one may expect logarithmic variations only (or even no dependence for $\left.|t| \leq \Lambda^{2} Q C D\right)$. With the limited experimentally accessible range in $t$, this should be a reasonable first approximation.

The evaluation of Eq. (3) yields the $\sigma_{p} p \rightarrow j j\left(M_{X}\right)$ shown in Fig. 2a for the above two gluon distributions and for two values of a minimum jet transverse momentum of 8 and $16 \mathrm{GeV}$. To illustrate the expected distribution of jets in the laboratory frame when $V s=630 \mathrm{GeV}$, we note that the boost from the $\mathrm{cms}$ of system $X$ to the laboratory is completely specified by the mass, $\mathrm{M}_{X}$. Results of a Monte Carlo event simulation according to Eqs. $(3,4)$ are shown in Fig. 3 , which is a scatter-plot of laboratory angle versus energy of jets with $p_{t} \geq 8 \mathrm{GeV}$. Due to the boost involved, the gluon jet from the slow pomeron emerges at large laboratory angles in a typical configuration, whereas the parton jet from the fast proton emerges at small angles, as illustrated in Fig. 1c.

The gluon distribution in the pomeron is experimentally measurable because a change in its shape shifts the parton-parton cms with respect to the $X \mathrm{cms}$. To illustrate this, we show as the solid curve in Fig. 4, the longitudinal momentum distribution for the jets in Fig. $3 b$, transformed to the $X \mathrm{cms}$. The dashed curve shows the corresponding distribution for the soft structure function of Eq. (5). In particular we note that since the softer function more nearly resembles the average structure function of the proton, there is a near coincidence of these two centers-of-mass and hence an almost symmetric distribution in Fig. 4. As expected, the hard structure function gives an asymmetric distribution in this frame. Experimental data can be plotted in this way for bins in $M_{X}$ and $t$ in order to determine the effective structure function. Alternatively, the variable $x_{1}=2 \cdot p_{t} / M_{X}$ can be used for this study. 
In order to evaluate Eq. (2) we approximate the high energy single diffractive cross-section measured at the SPS collider [1] by $\mathrm{d}^{2} \sigma / \mathrm{dM}^{2} \mathrm{dt}=6.8 / \mathrm{M}^{2} \cdot\left\{\mathrm{e}^{5.6 \cdot t}+0.04 \cdot \mathrm{e}^{2 \cdot t}\right\} \mathrm{mb} / \mathrm{GeV}^{4}$, where the constant 6.8 includes both reaction (1) and its charge conjugate. Introducing an experimental acceptance funtion $A\left(M_{X}, t\right)$ for the quasi-elastically scattered $\bar{p}(p)$, Eq. (2) becomes

$\frac{d^{2} \sigma_{j i}}{d t d M_{x}^{2}}=\frac{6.8}{M_{x}^{2}} \cdot\left\{e^{5.6 t}+0.04 e^{2 t}\right\} \cdot \frac{\sigma_{p \mathbb{P} \rightarrow j i}}{\sigma_{p \mathbb{P} \rightarrow X}} \cdot A\left(t, M_{x}\right)$

The concept of a proton-pomeron total cross-section, $\sigma_{p P \rightarrow X^{\prime}}$ can be defined and extracted from a Regge analysis of elastic scattering and single diffractive dissociation [8]. Although $\sigma_{p} p \rightarrow X$ depends somewhat on the variables $M_{X}$ and $t$, we approximate it below with a constant, $\sigma_{\mathrm{p} P \rightarrow X}=1 \mathrm{mb}$.

Using Eq. (3) for $\sigma_{p p \rightarrow j j}$ and integrating over all $t$, Eq. (6) yields the mass dependence shown in Fig. $2 b$ for the two structure functions of Eqs. $(4,5)$. The upper curves show the predicted cross-sections assuming complete experimental acceptance, i.e. $A\left(M_{X}, t\right)=1$, while the lower curves are calculated using a typical acceptance funtion ${ }^{*}$ ). The $M_{X}$ dependence shown in Fig. $2 b$ reflects the fact that the decrease in diffractive production with increasing $M_{X}$ is compensated by the $M_{X}$ dependence of the hard scattering cross-section $\sigma_{p P \rightarrow j j}$. Table I contains the total cross-sections at SPS collider and Fermilab Tevatron energies. As is usual with high- $p_{t}$ processes, the crosssections depend strongly on the lower $p_{t}$ cutoff.

We note that there may be other types of diffractive processes that yield high-p $p_{t}$ jets. In the model of ref. [9], the two gluons in the pomeron are both absorbed in a hard interaction giving rise to two jets that may appear at large angles, but without spectator jets along the beam direction. This mechanism also results in a different $M_{X}$ dependence. A coherent excitation of heavy flavours [10] with weak decays into lighter quark jets could have a cross-section with magnitude similar to ours, but would typically give more than two jets at large $p_{t}$. The semileptonic decay mode could be used to identify this heavy flavour process.

*) We approximate this acceptance by $A(t)=0.2 \cdot e^{0.5 t}$ with upper and lower cut-offs in $t$ at -0.6 and $-2.0 \mathrm{GeV}^{2}$ respectively. 
It should also be possible to study pomeron-pomeron collisions producing a central hadronic system if both the $p$ and $\bar{p}$ are tagged as being quasi-elastically scattered. This will be particularly interesting at a future $\mathrm{TeV}$ collider where large mass central systems are possible. One could further speculate whether there is a pomeron component in the proton, given by an effective structure function. If so, it can conceivably be probed in other interactions as well. One interesting possibility would be with an electromagnetic current as e.g. in electron scattering at HERA energies where the photon-gluon fusion process could occur. Of course, the photon could also directly couple to a quark sea in the pomeron. The advantage would be to have a well understood probe to study the point-like structure of the pomeron with very high resolving power, $\mathrm{Q}^{2}$. Again, the experimental signature would be clear; a quasi-elastically scattered proton "(going down the beam pipe) well separated from the remaining hadronic system.

We are grateful to P.V. Landshoff and G. Veneziano for several helpful discussions. 


\section{REFERENCES}

[1] M. Bozzo et al. (UA4 collaboration), Phys. Lett. 136B (1984) 217

[2] For recent reviews of diffractive interactions see, for example U. Amaldi, M. Jacob, G. Matthiae, Ann. Rev. Nucl. Sci. 26 (1976) 385

K. Goulianos, Phys. Rep. 101 (1983) 169

[3] A. Donnachie, P.V. Landshoff, Nucl. Phys. B244 (1984) 322

[4] S. Nussinov, Phys. Rev. Lett. 34 (1975) 1286, Phys. Rev. D14 (1976) 246

F.E. Low, Phys. Rev. D12 (1975) 163

[5] R. Cutlers, D. Sivers, Phys. Rev. D17 (1978) 196

R. Horgan, M. Jacob, Nucl. Phys. B179 (1981) 441

[6] M. Gluck, E. Hoffman, E. Reya, Z. Phys. C13 (1982) 119

[7] For a recent theoretical review of the photon structure function see, for example:

D.W. Duke, proceedings of the 5th international workshop on photon-photon collisions, Ed. Ch. Berger, Springer-Verlag 1983.

[8] K.A. Ter-Martirosyan, Phys. Lett. 44B (1973) 179

A.B. Kaidalov, K.A. Ter-Martirosyan, Nucl. Phys. B75 (1974) 471

D.P. Roy, R.G. Roberts, Nucl. Phys. B77 (1974) 240

See also Amaldi et al, in ref. [2].

[9] J. Randa, Phys. Rev. D22 (1980) 1583

T.A. DeGrand, J. Randa, Phys. Lett. $110 B$ (1982) 484

[10] R. Horgan, M. Jacob, Phys. Lett. 107B (1981) 395 


\section{FIGURE CAPTIONS}

Figure 1: a) Single pomeron exchange producing a heavy hadronic system, $X$, in the interaction $\bar{p}+p \rightarrow \bar{p}+X$ b) The hard parton-parton scattering subprocess in the pomeron-proton system, i.e. the lower vertex (or "blob") in a). c) The topology of the scattering process as seen in the overall $\bar{p} p$ cms; $\bar{p}_{f}$ is the quasi-elastically scattered antiproton, $J$ denotes the jets from the hard scattering and $\mathrm{S}$ denotes the spectator jets. Double arrows are the jets originating from the pomeron, while simple arrows are those from the proton.

Figure 2: a) Cross-section of the hard proton-pomeron scattering as a function of its invariant mass, $M_{X^{\prime}}$ for two values of the minimum $p_{t}$ required for the jets, using the hard (full lines) and soft (dashed lines) gluon distributions of the pomeron, eq. (4) and (5) respectively. b) Dependence on $M_{X}$ for the complete process $p+\bar{p} \rightarrow \bar{p}+X$, where $X$ contains two jets with $p_{t} \geq 8 \mathrm{GeV}$. The upper curves are for full acceptance in $t$ while the lower curves are for a typical experimental acceptance of the quasi-elastic $\bar{p}(p)$. Dashed and full lines as in a).

Figure 3: Scatterplot of angle versus energy for jets with $p_{t} \geq 8 \mathrm{GeV}$ in the laboratory frame ( $\bar{p} \mathrm{cms}$ ) for two values of the diffractive mass, $M_{X}$, using the hard gluon structure funtion of eq. (4). $\theta^{*}$ is the angle with respect to the pomeron momentum in the protonpomeron $\mathrm{cms}$.

Figure 4: Distribution in scaled longitudinal momentum, $x_{F}=2 \cdot p_{\mathbb{U}}^{*} / M_{X}$, of the two jets in the proton-pomeron cms for the hard (full line) and soft (dashed line) gluon distribution in the pomeron. 
Total cross-sections $\left(\mathrm{cm}^{2}\right)$

\begin{tabular}{ccccc}
\hline $\begin{array}{c}\text { Structure function } \\
\mathrm{xG}(\mathrm{x})\end{array}$ & \multicolumn{2}{c}{$\sqrt{\mathrm{s}}=630 \mathrm{GeV}$} & \multicolumn{2}{c}{$\sqrt{\mathrm{s}}=2 \mathrm{TeV}$} \\
& $\mathrm{p}_{\perp}>8 \mathrm{GeV}$ & $\mathrm{p}_{\perp}>16 \mathrm{GeV}$ & $\mathrm{p}_{\perp}>16 \mathrm{GeV}$ & $\mathrm{p}_{\perp}>32 \mathrm{GeV}$ \\
\hline $6 \times(1-\mathrm{x})^{1}$ & $6 \cdot 10^{-29}$ & $2 \cdot 10^{-30}$ & $3 \cdot 10^{-29}$ & $1 \cdot 10^{-29}$ \\
& $\left(5 \cdot 10^{-31}\right) *$ & $\left(2 \cdot 10^{-32}\right) *$ & & \\
$6(1-\mathrm{x})^{5}$ & $4 \cdot 10^{-29}$ & $6 \cdot 10^{-31}$ & $2 \cdot 10^{-29}$ & $6 \cdot 10^{-30}$ \\
& $\left(3 \cdot 10^{-31}\right) *$ & $\left(5 \cdot 10^{-33}\right) *$ & & \\
\hline
\end{tabular}

* These cross-sections include a typical experimental acceptance of the recoil $p(\bar{p})$ as discussed in the text. 
a)

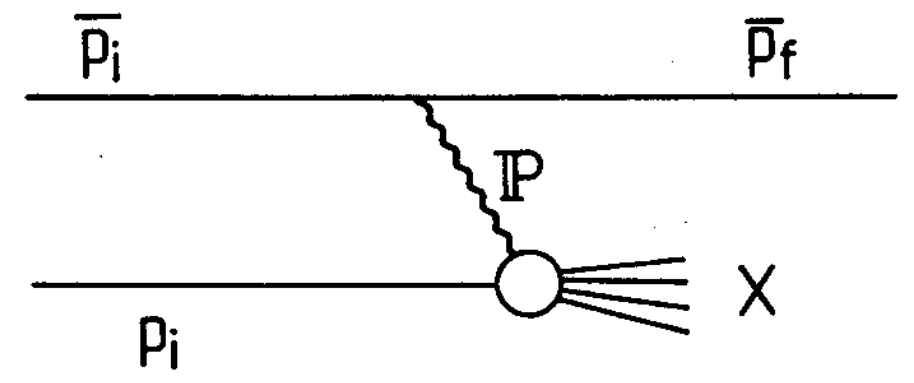

b)

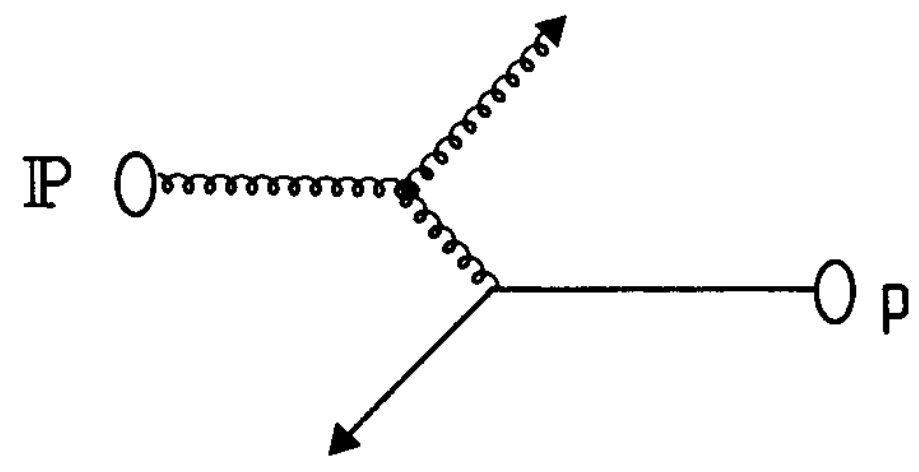

c)

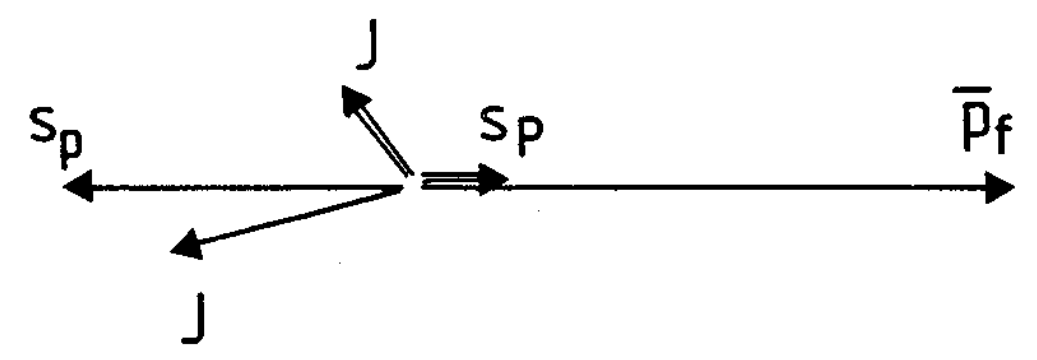

Fig. 1 


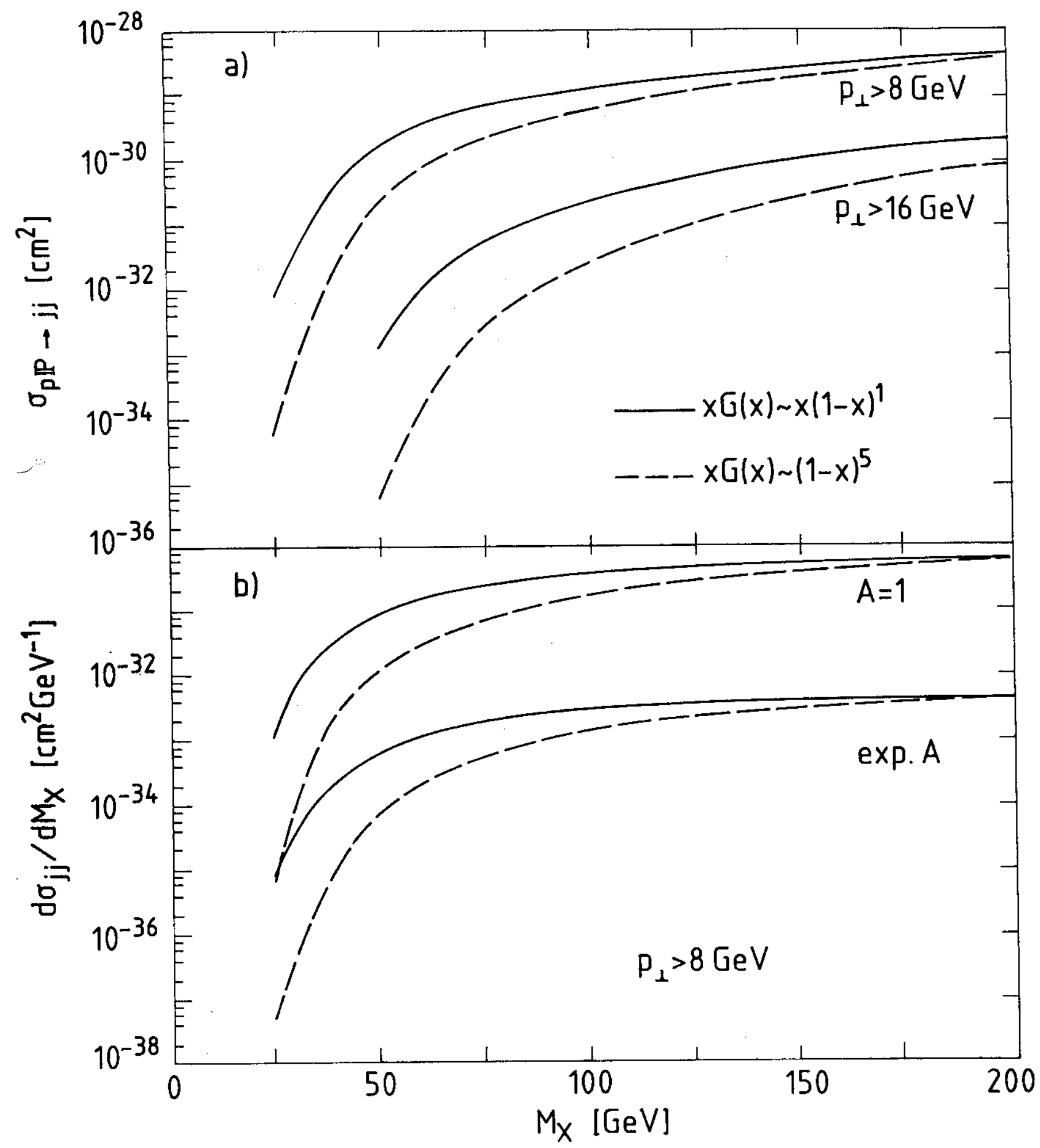

Fig. 2 


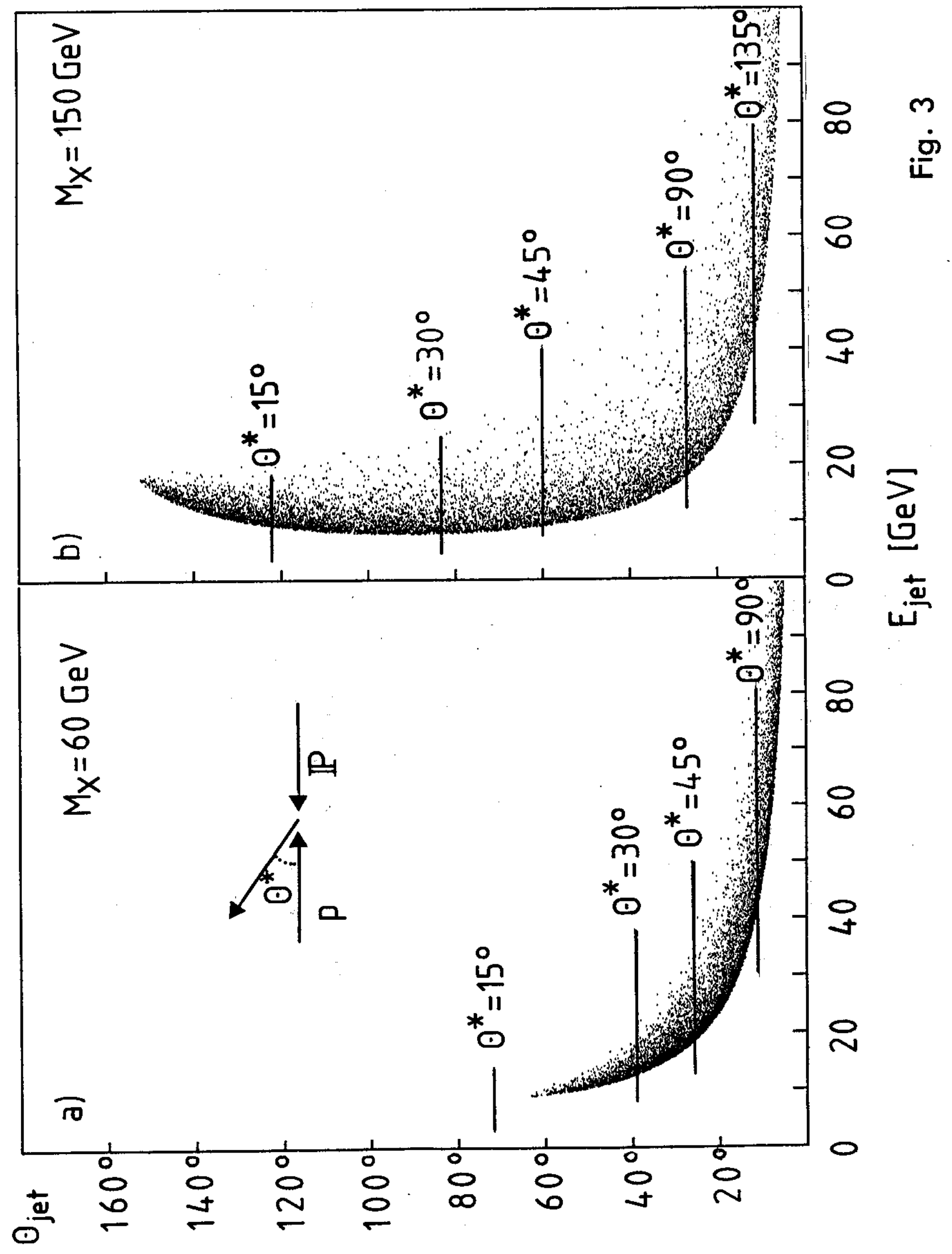




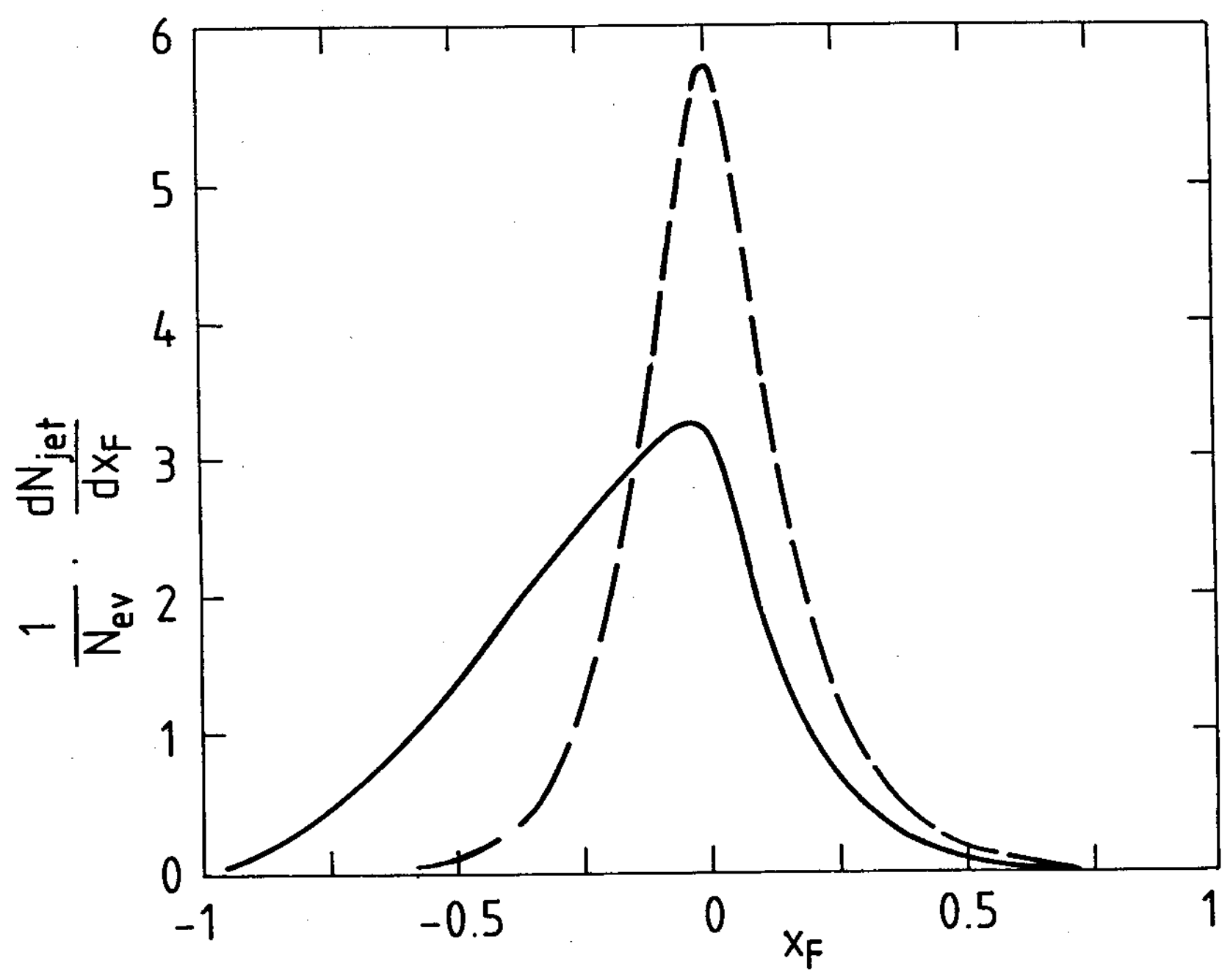

Fig. 4 